\title{
Randomized response surface pathway design with odd response outcomes in a Latin Square designed study
}

\section{Trond Holand'}

Kristian Ellingsen ${ }^{2}$

Sagita Dewi ${ }^{1,3}$

Stig Larsen'

'Centre for Epidemiology and Biostatistics, Department of Production Animal Clinical Sciences, Faculty of Veterinary Science, Norwegian University of Life Science, ${ }^{2}$ Norwegian Veterinary Institute, Oslo, Norway; ${ }^{3}$ Bali Indera Hospital, Government of Bali Province, Denpasar, Bali, Indonesia
Correspondence: Stig Larsen Faculty of Veterinary Science, Department of Production Animal Clinical Sciences, Centre for Epidemiology and Biostatistics, Norwegian University of Life Science, Ullevaalsveien 72, N-0354 Oslo, Norway Tel +474l 326325

Email stig.larsen@nmbu.no
This article was published in the following Dove Press journal:

Open Access Journal of Clinical Trials

14 September 2017

Number of times this article has been viewed

Background: Response surface pathway (RSP) design has been recently developed and described for dose-finding studies. The aim of this paper was to introduce and demonstrate additional procedures to strengthen and generalize the design and combine RSP with classical study design.

Materials and methods: Nine bull calves and six heifer calves were included in an unbalanced $2 \times 2$ Latin Square (LSQ) designed study with large and small aperture bottle teats. The two LSQ sequences were performed with independent randomized three-level between-patient RSP design with an odd number of response classifications. The milk temperature window was $8^{\circ} \mathrm{C}-38^{\circ} \mathrm{C}$ with a mid temperature of $23^{\circ} \mathrm{C}$. X-rays of the abdominal cavity were taken before, during, and immediately after intake of milk and recorded as "milk", "trace", or "no milk". Based on the results of the first design level, the milk temperatures for five calves in the second design level were obtained by a randomization procedure. A similar procedure was performed for seven calves in the third design level. Adjustment of the dose from one design level to the next was based on a k-adjustment factor estimated to ensure coverage of the entire predefined dose window.

Results: Starting with a low number of subjects and increasing this number with increasing design levels reduces the sample size without reducing the power. The suggested randomization procedure worked as expected. No milk in Rumen was recorded and minimum milk temperature (MMT) was estimated to be $\leq 8^{\circ} \mathrm{C}$ for both teats. The odd number of response categories increases the flexibility of RSP, enabling category redefinition in hindsight from "trace" to "uncertain". After category redefinition, MMT for large bottle teat was estimated to be $14.3^{\circ} \mathrm{C}$ (95\% confidence interval: $8.3-20.3^{\circ} \mathrm{C}$ ), but $8^{\circ} \mathrm{C}$ for small.

Conclusion: The suggested changes and additional procedures increase the strength and flexibility of the RSP design.

Keywords: k-adjustment factor, Latin Square design, patient reduction in clinical trials, randomized response surface pathway design

\section{Background}

During the first few weeks of life, milk is the primary source of nutrition for the dairy calf. Over the milk feeding period, the calf has an anatomical feature called the esophageal groove or sulcus reticuli. The groove, when activated, turns into a tube allowing the milk to pass by the reticulorumen straight into the abomasum where the milk is digested. ${ }^{1}$ Activation of the reticular groove is caused by many factors, including the smell and taste of milk, milk temperature, sucking behavior, and body posture while drinking. ${ }^{2}$ If the esophageal groove is not properly closed, milk can enter Rumen, the 
first chamber of the ruminant stomach, rather than the abomasum. In very young animals, this may not pose a problem as the Rumen is emptied into the abomasum within a few hours. ${ }^{3}$ For older calves with ruminal development, milk in the Rumen is undesirable as it may affect the ruminal $\mathrm{pH}$ and cause indigestion, diarrhea, and reduced growth. ${ }^{4}$ To ensure efficient and complete closure of the reticular groove, it is generally recommended to feed dairy calves with warm milk at $38^{\circ} \mathrm{C}$ from a small aperture teat. Practical experience shows that this is not always followed as farmers often are found to save time during manual feeding by cutting off the convex tip of the rubber teat. This increases the aperture from a few millimeters to $2 \mathrm{~cm}$ and drastically increases the drinking speed. Additionally, farmers often feed milk that has been insufficiently heated or not heated at all. The aim of this calf study was to estimate minimum milk temperature (MMT) for both small and large aperture bottle teats.

Choice of study design for an interventional uncontrolled clinical trial (UCCT) and controlled clinical trial (CCT) constitutes a challenge for researchers worldwide. ${ }^{5,6}$ Ethical and scientific conduct, coupled with scarce resources, requires choice of a study design that combines a minimum of included patients while producing as strong statistical results as possible. These opposing goals make if difficult to choose an efficient study capable of handling different combinations of interventions and measurable clinical outcomes in a challenging landscape critical. "Response surface pathway" (RSP) is a study design methodology based on combining classical up-and-down procedure with common response surface methodology. ${ }^{7-10}$ The overriding aim for further development of the RSP methodology is clinical documentation of a study design capable of reducing need for unnecessary patient inclusion without reducing accuracy. Earlier UCCT using RSP methodology has focused on documentation in clinical trials without inclusion of randomization procedures, odd number of outcomes, and combinations with other classical study designs. ${ }^{11}$

Introduction of an increasing number of included patients from one design level to the next is one way of identifying a more targeted and efficient pathway toward the area of clinical interest. For many clinicians, the methodological aim behind increasing patients with increasing design level is intuitively logical in a practical trial setting compared to the traditional equal number of included patients in each consecutive study level approach. ${ }^{11}$ The benefit of the patient-increase procedure has been shown to optimize study design goals in lethal dose $(\mathrm{LD})_{50}$ studies in a laboratory setting, but has not yet been tested in dose-finding studies. However, replacement of the equal number strategy with patient-increase procedure introduces the need for randomization procedure. Randomization is one of the cornerstones of the randomized controlled trial (RCT) and a true random allocation procedure should be used. ${ }^{12,13}$ Is it possible to construct an optimal randomization procedure allocating patients to different doses at one RSP dose level based on the outcome from the previous study?

The RSP method has previously been developed for outcome variables divided into an equal number of outcome categories. ${ }^{10}$ This is based on earlier use in $\mathrm{LD}_{50}$ studies, where the mid value is the goal, and an odd number of patients at each design level is demanded. This would not be necessary with odd outcome categories. The RSP method can be developed further from an equal to an odd number of outcome categories to unlock versatility in a practical clinical research setting. Further development of the RSP method by combining it with other study designs has been identified as another alternative to increase strength, versatility, and practical usability of this methodology. Combining RSP design with other traditional designs, like parallel group or Latin Square (LSQ), could increase the applicability of RSP.

The aim of this paper is to introduce and demonstrate RSP with increasing patient number along with increasing design levels, randomization procedure between design levels, odd number of study outcomes, and RSP used in an LSQ designed study to estimate MMT for young calves using small and large aperture bottle teats.

\section{Materials and methods}

Nine bull calves and six heifer calves were used in the development of this randomized RSP designed study with odd response classification. The animals were classified with at least sufficient feeding condition, very good general health condition, and no abnormalities. The mean age of the calves was 18.5 days (range: 9-27 days) with a mean body weight of $54.9 \mathrm{~kg}$ (range: $45.5-71.0 \mathrm{~kg}$ ). After being evaluated by the Norwegian Animal Research Authority, the study was exempt from having to apply for approval because the participating animals served as a model in a non-invasive feeding experiment with no expected side effects.

\section{Study performance}

The study is a randomized single-center study with an unbalanced $2 \times 2$ LSQ design. Each calf participated twice and was offered $4 \mathrm{~L}$ of whole milk per trial, once via a small (S) and once via a large (L) aperture bottle teat. In accordance with LSQ, the calves randomly received the milk in the sequence $\mathrm{S}-\mathrm{L}$ or L-S. To control the influence of a possible sequence 
effect and to allow potential milk in the Rumen to be passed on, washout period of 2 days between the two feedings was included.

A between-patient RSP design with milk temperature as the interventional variable and milk in Rumen as response was independently used for the small and the large aperture teat arms. ${ }^{10}$ The milk temperature with the given bottle teat to be used at one design level is based on the results obtained on the previous design level. The start temperature on the first design level was $23^{\circ} \mathrm{C}$ with a lower limit of $8^{\circ} \mathrm{C}$ and an upper limit of $38^{\circ} \mathrm{C}$. Each calf was offered $4 \mathrm{~L}$ of milk with the given temperature. Milk consumption was recorded in liters, and the duration of the feeding in seconds. X-rays of the abdominal cavity were taken before, during, and immediately after feeding, and milk in the Rumen was recorded as "milk", "trace", or "no milk". If no difficulties were encountered, the second feeding with the other bottle teat could start. Adverse events were registered the following 2 days.

\section{Ethics approval and consent to participate}

Norwegian national authority-approved protocols were followed with regard to the animal welfare during our study following our publication ethics standard. Consent for publication is not applicable.

\section{RSP design}

The RSP methodology has previously been presented both for within and between different groups of patients with one interventional- and one response variable. ${ }^{10}$ In order to capture, utilize, and adjust according to accumulated clinical information during the study, the number of included subjects is reduced to a minimum in the first design level and increases with increasing level. In the between-patient RSP design, dimension groups of three patients are allocated into sequences with the same number of patients as the number of levels in the design. In this between-patient model, the RSP design consists of $n$ levels and $r$ independent patient sequences. The first patient in the sequence receives the predefined starting dose. ${ }^{10,14}$ The response from this patient determines the dose for the second patient in the same sequence. In general, the response from the patient at design level $\mathrm{i}(1 \leq \mathrm{i} \leq$ n) determines the dose for the patient at the next design level $\mathrm{i}+1$. The sequences develop a random walk and describe a pathway with equal number of patients at each design level.

Development of the methodology in laboratory animals and simulations demonstrated that this equal number strategy is not an optimal solution. ${ }^{11}$ By starting with a low number of patients at the first design level and increasing this number with increased level, the sample size reduces without reduction in accuracy. This is possible in the between-patient RSP design using randomization.

\section{Randomized between-patient RSP design}

To optimize the RSP model, the number of patients reduces to a minimum of $\geq 2$ in the first design level and increases with increasing level. The previously recommended patient-increase procedure starts with three patients at level 1 increasing to five, seven, nine etc., at level 2 and upward. ${ }^{10}$ The three calves allocated to the first design level receive $4 \mathrm{~L}$ of milk with a temperature of $23^{\circ} \mathrm{C}$. Assume that $\mathrm{a}_{1}$ represents the number of calves on the first design level indicating milk temperature $A_{1}$ for the second design level, $a_{2}$ pointing out milk temperature $\mathrm{A}_{2}$, and $\mathrm{a}_{3}$ pointing out milk temperature $\mathrm{A}_{3}$ (Figure 1). In general, a weighted randomization in ratio $\left(a_{1}: a_{2}: a_{3}\right)$ will be appear. If two doses are equal $\left(\mathrm{a}_{1}=\mathrm{a}_{2}\right)$ and and the third dose is deviating, the calf on the second level will be weighted randomized $(2: 1)$. This means that the probability for a calf to be allocated to milk temperature $A_{1}=A_{2}$ is $2 / 3$ and $1 / 3$ for milk temperature $\mathrm{A}_{3}$ (Table 1).

Based on the milk in Rumen classification obtained in the five calves on the second design level, theoretically five new milk temperatures $\left(\mathrm{B}_{1} ; \mathrm{B}_{2} ; \mathrm{B}_{3} ; \mathrm{B}_{4} ; \mathrm{B}_{5}\right)$ allocate to the seven patients in the third design level. Assume that $b_{1}$ calf on the second design level calculated by the RSP procedure to receive intervention $\mathrm{B}_{1}, \mathrm{~b}_{2}$ to receive dose $\mathrm{B}_{2}, \mathrm{~b}_{3}$ to receive dose $\mathrm{B}_{3}, \mathrm{~b}_{4}$ to receive dose $\mathrm{B}_{4}$, and $\mathrm{b}_{5}$ to receive dose $\mathrm{B}_{5}$. The milk temperature to be used on the third design level for the seven new calves will then be allocated by weighted complete randomization $\left(b_{1}, b_{2}, b_{3}, b_{4}, b_{5}\right)$ following the same procedure as explained for the second design level. By replacing the previously described sequence-procedure in allocation to design levels with this randomization procedure, while keeping the other part of the RSP, a randomized between-patient RSP designed RCT is introduced.

\section{Combination of randomization results in a $2 \times 2$ LSQ design}

Let N1, N2, and N3 denote the RSP-calculated milk temperature for the small teat of the bottle opening and M1, M2, and M3 denote the same for the large teat. The three calves in level 1 indicate the three milk temperature combinations: "N1-M1", "N2-M2", and "N3-M3".

These three milk temperature combinations for small and large teats of the bottle openings will be used as the basis 


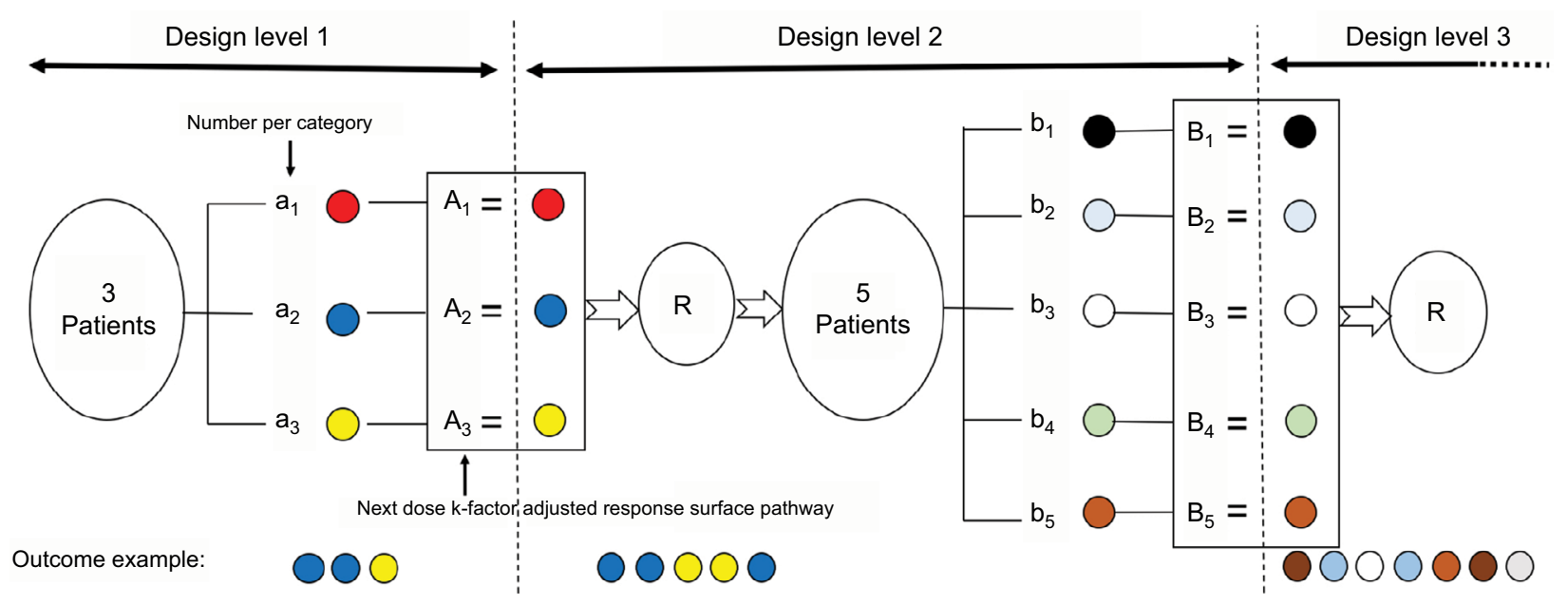

Figure I Randomization procedure.

Notes: Small letters $a_{1-3}$ and $b_{1-5}$ indicate the numbers within each outcome category. $A_{1-3}$ and $B_{1-5}$ represent the dosage to be used in the next design level. The three possible clinical outcomes from the first patients in one design level point to the next five k-factor-adjusted doses in the next design level. "R" represents the randomization from one design level to the next.

Table I Randomization of the milk temperature for the next design level based upon the outcome in the previous level for given bottle teats

\begin{tabular}{|c|c|c|c|}
\hline Design level & Milk temperature $\left({ }^{\circ} \mathrm{C}\right)$ & Randomization & Dosage used in the next design level $\left({ }^{\circ} \mathrm{C}\right)$ \\
\hline Design level I $(n=3)$ & 23 & None & $\begin{array}{l}a_{1} \text { patient randomizes to } A_{1} \\
a_{2} \text { patient randomizes to } A_{2} \\
a_{3} \text { patient randomizes to } A_{3}\end{array}$ \\
\hline Design level $2(n=5)$ & $\begin{array}{l}A_{1} \\
A_{2} \\
A_{3}\end{array}$ & $a_{1}: a_{2}: a_{3}$ & $\begin{array}{l}b_{1} \text { patients randomizes to } B_{1} \\
b_{2} \text { patients randomizes to } B_{2} \\
b_{3} \text { patients randomizes to } B_{3} \\
b_{4} \text { patients randomizes to } B_{4} \\
b_{5} \text { patients randomizes to } B_{5}\end{array}$ \\
\hline Design level $3(n=7)$ & $\begin{array}{l}\mathrm{B}_{1} \\
\mathrm{~B}_{2} \\
\mathrm{~B}_{3} \\
\mathrm{~B}_{4} \\
\mathrm{~B}_{5}\end{array}$ & $b_{1}: b_{2}: b_{3}: b_{4}: b_{5}$ & $\begin{array}{l}c_{1} \text { patients randomizes to } C_{1} \\
c_{2} \text { patients randomizes to } C_{2} \\
c_{3} \text { patients randomizes to } C_{3} \\
c_{4} \text { patients randomizes to } C_{4} \\
c_{5} \text { patients randomizes to } C_{5} \\
c_{6} \text { patients randomizes to } C_{6} \\
c_{7} \text { patients randomizes to } C_{7}\end{array}$ \\
\hline
\end{tabular}

Note: Capital letters show the milk temperature category and small letters indicate the number of subjects within each category.

for allocation of the five calves participating in the second design level.

A similar procedure is performed for the third design level. Let N4-N8 denote the RSP-calculated milk temperature recommended for the small teat on the bottle, while M4-M8 for large bottle teat on the third design level. The basis for randomization of the seven calves in design level 3 will be "N4-M4", "N5-M5", "N6-M6", "N7-M7", and "N8-M8". This procedure builds on and considers the dependency within calves.

\section{Dose adjustment procedure in RSP design}

Let $m$ denote the starting dose, $m_{i}$ the dose at design level $i$, and $\mathrm{k}$ the dose adjustment factor. The dose at design level $\mathrm{i}$ is given by equation (1). Let $\mathrm{D}_{\mathrm{u}}$ denote the upper limit of the interventional variable and $\mathrm{n}$ the number of design levels, then $\mathrm{D}_{\mathrm{u}}$ is given as the sum of a geometric series in equation (2). ${ }^{15}$

$$
\begin{aligned}
& m_{i}=m_{i-1} \pm \frac{m}{k^{i-1}} \\
& \mathrm{D}_{\mathrm{u}}=\sum_{\mathrm{i}=1}^{\mathrm{n}} \mathrm{m}(1 / \mathrm{k})^{i-1} \\
& D_{U}=\frac{m\left(k^{n}-1\right)}{\left(k^{n}-k^{n-1}\right)}
\end{aligned}
$$

With known upper limit of the interventional variable, the starting value $\mathrm{m}$, and design level $\mathrm{n}$, the $\mathrm{k}$-adjustment factor is calculated from equation (2). 


\section{Escalation and de-escalation procedure}

The response variable is multinomial with unequal number of categories denoted as $2 c+1$. Of these possible $2 c+1$ response values, one of the c's gives escalation, one c deescalation, and one remaining unchanged value of the interventional variable for the patients in the next design level. It may be convenient to use the mid value of the predefined dose window as the starting value denoted as $\mathrm{m}$. To ensure coverage of the dose window, a dose adjustment procedure is established.

Assume that the response variable has a sample space $\{1,2, \ldots 2 c, 2 c+1\}$, the median value will then be $c+1$. Let $\mathrm{i}$ represent the new design level, $\mathrm{j}$ the outcome from the previous dose level $\mathrm{i}-1$, and $\mathrm{h}$ the outcome from the dose level $\mathrm{i}-1$. For calculation of the first dose level $\mathrm{h} \equiv 1$ per definition. Let $\mathrm{m}_{1}=\mathrm{m}$. In this case, there are only three outcome values giving $\mathrm{c}=1$; then for design level $\mathrm{i} \geq 2$ the interventional value is given as:

$$
\begin{array}{ll}
m_{i}=m_{i-1}+m / k^{i+j+h-2 c-1} & ; j \leq c \& h \leq c \\
m_{i}=m_{i-1} & ; j=c+1 \& h \leq c \\
m_{i}=m_{i-1}-m / k^{i-j+h-2 c-1} & ; j>c+1 \& h \leq c \\
\hdashline m_{i}=m_{i-2}+m / k^{i-j+h-2 c} & ; j \leq c \& h=c+1 \\
m_{i}=m_{i-2} & ; j=c+1 \& h=c+1 \\
m_{i}=m_{i-2}-m / k^{i-j+h-2 c} & ; j>c+1 \& h=c+1 \\
-\cdots & ; j \leq c \& h>c+1 \\
m_{i}=m_{i-1}+m / k^{i-j+h-2 c-1} & ; j=c+1 \& h>c+1 \\
m_{i}=m_{i-1} & ; j>c \& h>c+1 \\
m_{i}=m_{i-1}-m / k^{i-j+h-2 c+1} &
\end{array}
$$

The equations above calculate the RSP dose adjustment procedure for the next design level.

\section{Statistical analysis}

The sample space of the milk temperature may be expressed as $\Omega_{\mathrm{T}}=\left\{\mathrm{D}_{\mathrm{L}} \leq \ldots \leq \mathrm{D}_{\mathrm{U}}\right\}$. Let $\mu_{\mathrm{d}}$ represent MMT for new born calves and assume $\mu_{\mathrm{d}}$ covers by $\Omega_{\mathrm{D}}$. Milk in Rumen is ordinal in interventional variable, and the probability increases monotonically over the interventional levels. Isotonic regression is the suggested model for analyzing the material. ${ }^{16}$ Continuously distributed variables are expressed as mean values, standard deviation (SD) in brackets, and $95 \%$ confidence interval. ${ }^{17}$

\section{Results}

At $23^{\circ} \mathrm{C}$ milk temperature in design level 1 , no milk was found in Rumen, neither with small nor large aperture teat of the bottle (Figure 2). However, one calf failed to consume more than two of the four prescribed liters with large teat. No randomization was needed, and all the five calves at design level 2 received $13^{\circ} \mathrm{C}$ milk with both teat sizes. Two calves were not able to consume the $4 \mathrm{~L}$ at this temperature using large teat. No milk in Rumen was obtained with neither of the two teat sizes and all the seven calves at design level 3 received the prescribed $4 \mathrm{~L}$ of milk with the temperature of $8^{\circ} \mathrm{C}$ (Figure 2). No milk in Rumen was detected in these seven calves neither with small nor large aperture teat of the bottle. However, two of the calves were not able to consume $>2$ and $3 \mathrm{~L}$ using small teat and one calf consumed $2.5 \mathrm{~L}$ with large teat. Without any adjustments for $<4 \mathrm{~L}$ milk consumption, the estimated MMT independent of teat size is $8^{\circ} \mathrm{C}$.

The classification used in the RSP procedure was only related to the X-ray findings without considering the deviation from the prescribed $4 \mathrm{~L}$ of milk. Four calves deviated from the prescribed amount when using large aperture teat and two calves when using small aperture teat. By expanding the classification "Trace" to "Uncertain", including the situation when a calf was not able to consume the prescribed $4 \mathrm{~L}$, the results change. For small teat, the pattern will be nearly unchanged except from design level 3. Instead of ending with seven calves without milk in Rumen at $8^{\circ} \mathrm{C}$, the results change to five calves without milk and two classified as "Uncertain" (Figure 3). This results in an estimated MMT of $8^{\circ} \mathrm{C}(95 \%$ confidence interval $[\mathrm{CI}]: 6.2-9.8^{\circ} \mathrm{C}$ ) for small teat.

For large teat, the change in results was more pronounced (Figure 4). In design level 1 with milk temperature of $23^{\circ} \mathrm{C}$, two calves were classified as "No milk" and one as "Uncertain". The randomization of five calves to the second design level will be $13^{\circ} \mathrm{C}$ and $23^{\circ} \mathrm{C}$ in a $2: 1$ ratio. The outcome with the largest probability will be three calves to $13^{\circ} \mathrm{C}$ and two to $23^{\circ} \mathrm{C}$. Assuming the two calves receiving milk with temperature $23^{\circ} \mathrm{C}$ at design 2 level will consume all $4 \mathrm{~L}$ without any milk in Rumen, this gives two times $21^{\circ} \mathrm{C}$ as contribution to the randomization for design level 3. Based on the study results, the probability for "No milk" with a temperature of $13^{\circ} \mathrm{C}$ is $3 / 5$ and $2 / 5$ for "Uncertain". The outcome with highest probability will be two "No milk" and one "Uncertain". The background for randomization to design level 3 will then be $\left(21^{\circ} \mathrm{C}, 21^{\circ} \mathrm{C}, 13^{\circ} \mathrm{C}, 8^{\circ} \mathrm{C}\right.$, and $8^{\circ} \mathrm{C}$ ). The expected number of calves randomized to $8^{\circ} \mathrm{C}$ and 


\begin{tabular}{|l|l|l|}
\hline Design level 1 & Design level 2 & Design level 3 \\
\hline
\end{tabular}

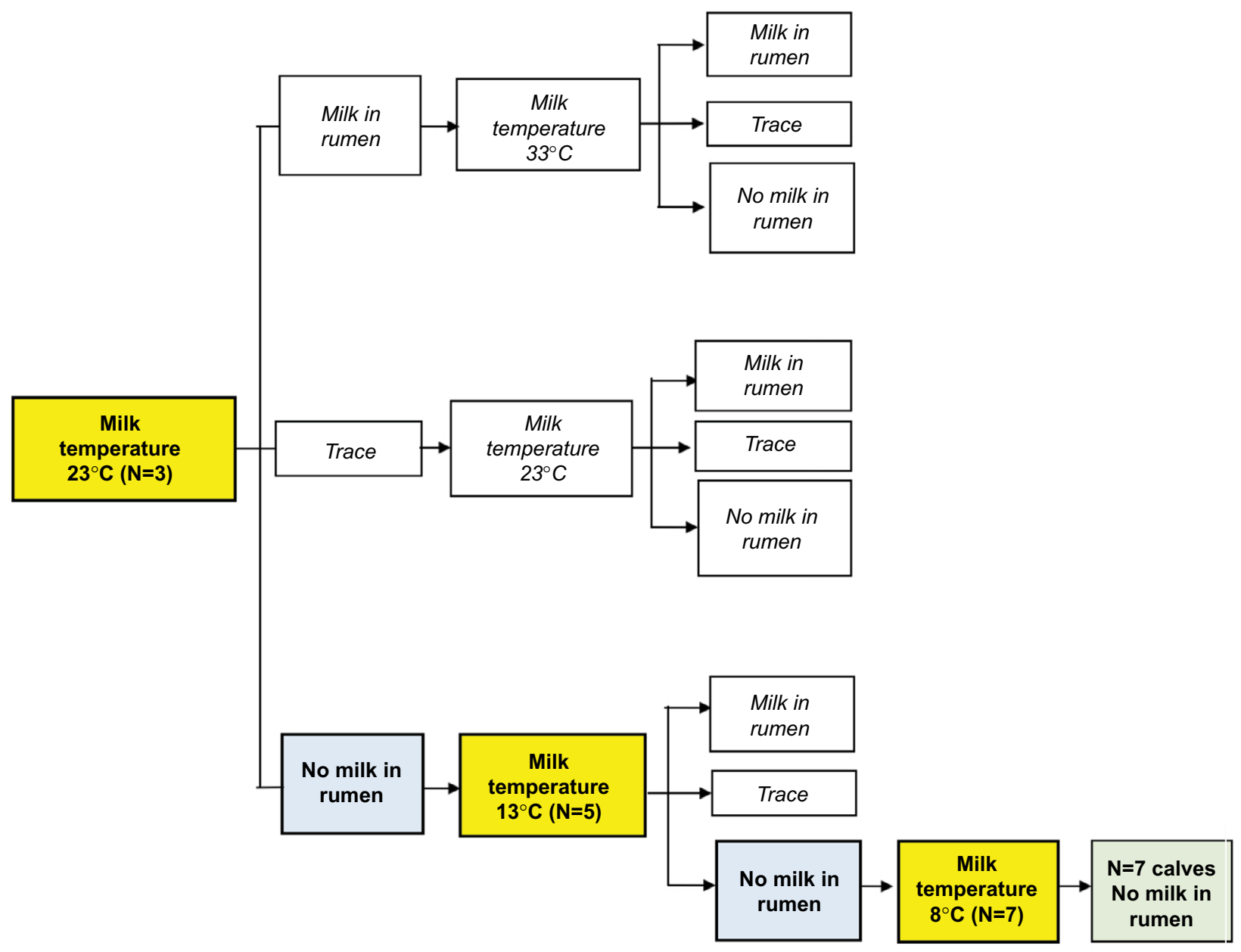

Figure 2 The obtained RSP pathway for the milk in rumen category (blue) based on milk temperature in ${ }^{\circ} \mathrm{C}$ (yellow) for both small and large bottle teats. Abbreviation: RSP, response surface pathway.

$21^{\circ} \mathrm{C}$ is 2.8 , and the expected number for $13^{\circ} \mathrm{C}$ is 1.4 . The most probable outcome of the randomization will be three calves receiving milk with the temperature $21^{\circ} \mathrm{C}$, one with milk temperature $13^{\circ} \mathrm{C}$, and three with milk temperature $8^{\circ} \mathrm{C}$. From the obtained study results, the most probable outcome with large teat is "No milk" for all seven calves for the given set of milk temperatures. This results in an estimated MMT of $14.3^{\circ} \mathrm{C}\left(95 \% \mathrm{CI}: 12.1-16.5^{\circ} \mathrm{C}\right)$ for large teat. In the LSQ analysis, the difference between small and large teat will reach the $5 \%$ significance level.

\section{Discussion}

Despite missing the targeted $4 \mathrm{~L}$ milk consumption in six of the 30 milk feedings, the study design was with sufficient accuracy able to predict likelihood of "milk" within an $8-38^{\circ} \mathrm{C}$ temperature window while indicating influence of small and large bottle teat sizes. The study design worked well, presented the opportunity to reinvestigate obtained results after reclassification of the mid outcome category, and demonstrated an efficient pathway toward the area of clinical interest.

RSP methodology in patient-related dose-response study has earlier used an equal number of included patients at each design level. Development of the concept in laboratory animals and computer simulations demonstrated that this is not an optimal solution. ${ }^{11}$ Starting with a low number of subjects and increasing this number with succeeding design levels reduces the sample size without reducing the accuracy of the estimates. This procedure is not possible in the within-patient situation, but can be implemented in the 


\begin{tabular}{|l|l|l|}
\hline Design level 1 & Design level 2 & Design level 3 \\
\hline
\end{tabular}

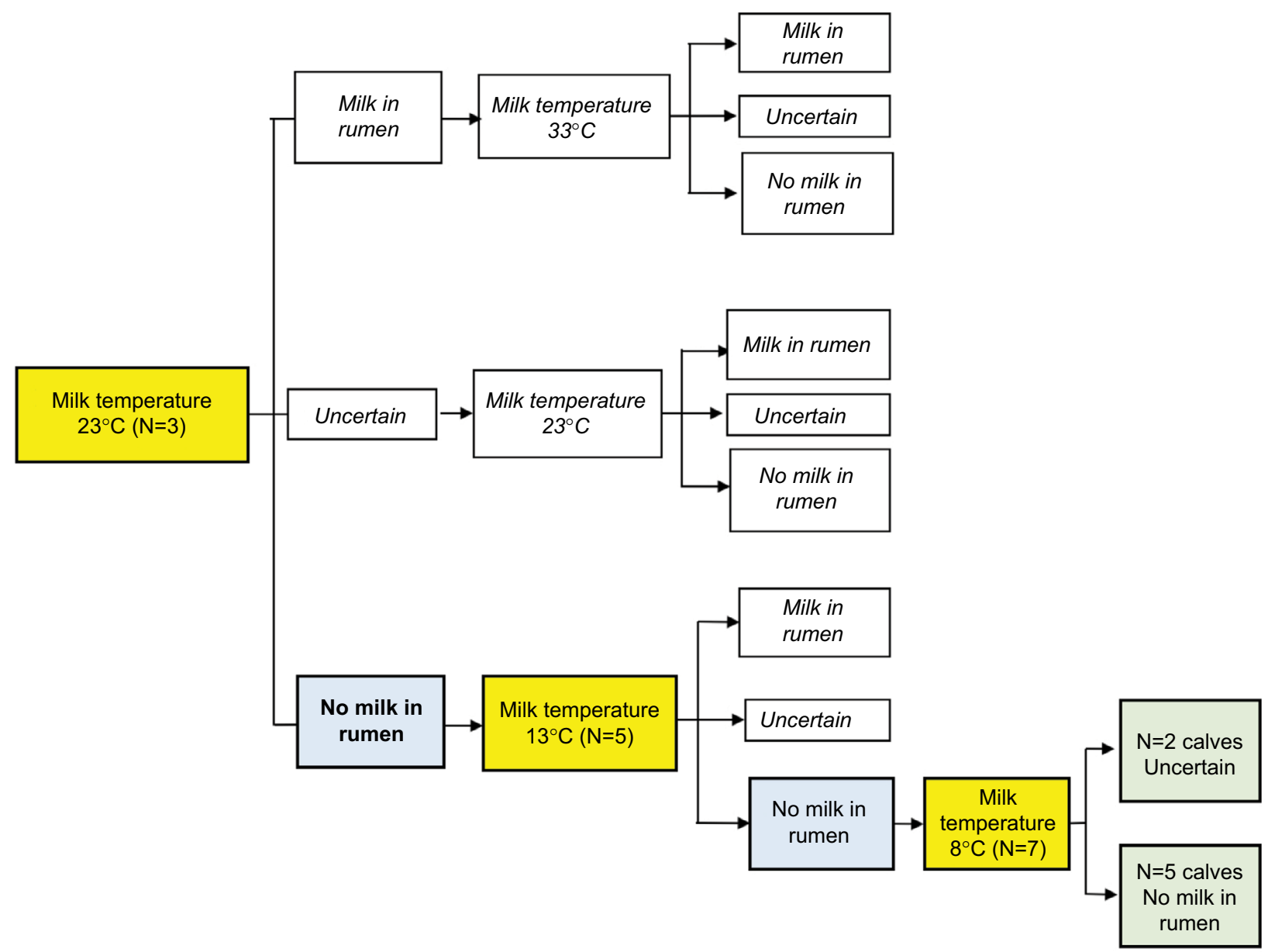

Figure 3 The adjusted RSP pathway for small teat after reclassifying "Trace" to "Uncertain".

Notes: The category (blue) is based on milk temperature in ${ }^{\circ} \mathrm{C}$ (yellow) for small bottle teat. The end results are given in green. "Trace" outcome category reclassification to "Uncertain" in the case of $<4 \mathrm{~L}$ milk consumption.

Abbreviation: RSP, response surface pathway.

between-patient RSP design by introducing a randomization procedure between design levels. Advantages from increasing the patient number, accompanied with an optimized randomization procedure between increasing design levels, have earlier been demonstrated.

Earlier studies using RSP methodology have used an odd number of patient-increase procedure starting with three patients at level 1 , increasing to $5,7,9$, and so on at level 2 and upward. In the present study, 15 calves were exposed twice and an odd number of three outcome categories recorded. Because of uncertainty regarding exact interpretation of X-ray findings, categories "Yes", "Trace", and "No" were chosen. The basic RSP model was introduced in an $\mathrm{LD}_{50}$ study requiring an odd number of mice in each design level, because $\mathrm{LD}_{50}$ was between the escalation and de-escalation pathways. ${ }^{11}$ The RSP methodology can, however, also be used in an even number of patient-increase procedure coupled with even or odd outcomes. Introducing odd outcomes can in this way unlock statistical strength and usability when an even number of included patients in each design level is feasible. Instead of an RSP sample size $3+5+7$ in design level 1, 2, and 3 including 15 subjects, a $2+4+6$ or $4+6+8$ sample size with odd outcomes for 12 or 18 subjects might be sufficient to maneuver the pathway to statistical and clinical relevance. Applied in the present trial, an RSP sample size of 12 subjects with the given outcomes, the same study results with no X-ray findings would have been reached. Introduction of an "Uncertain" category into study design can address 


\begin{tabular}{|l|l|l|}
\hline Design level 1 & Design level 2 & Design level 3 \\
\hline
\end{tabular}

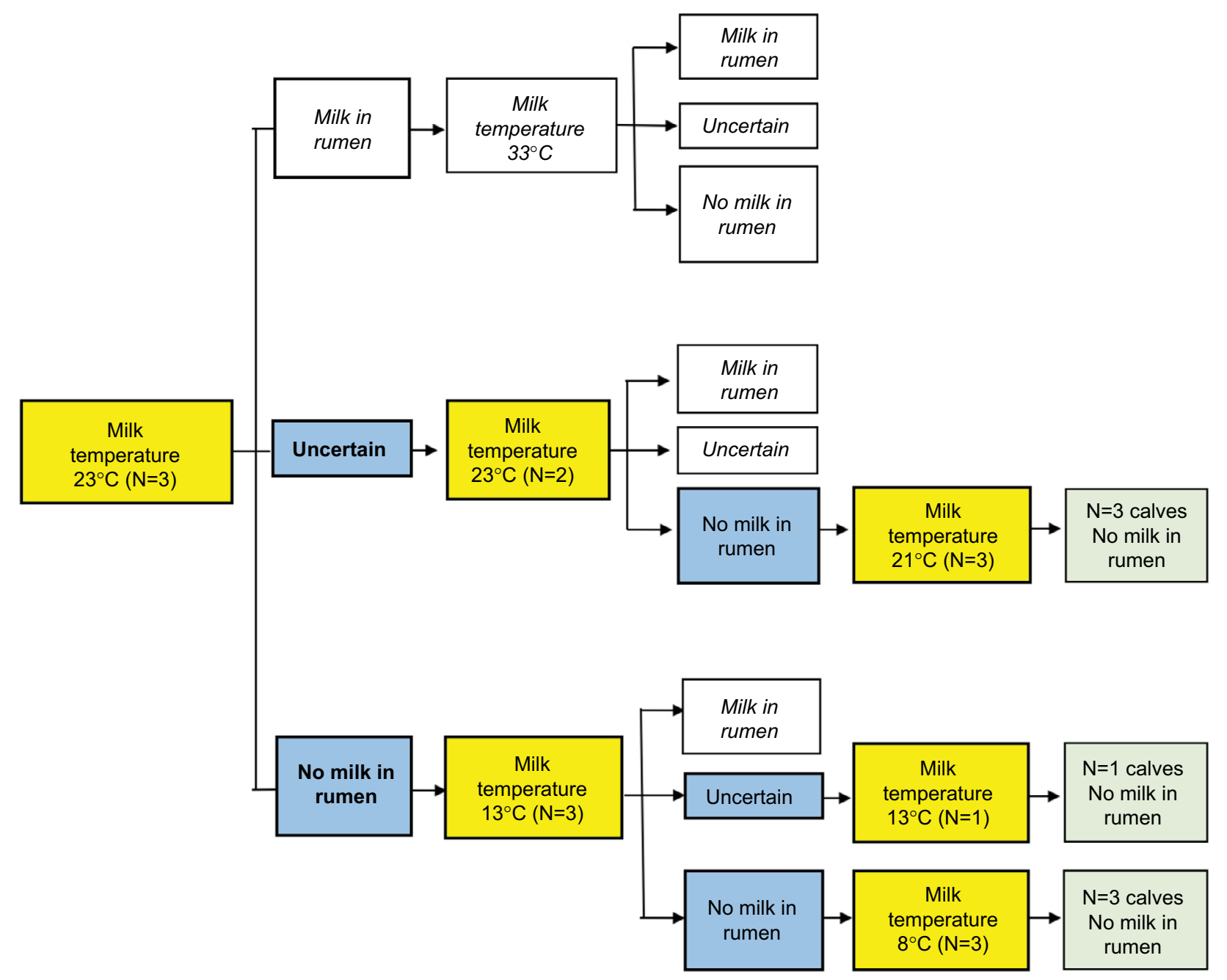

Figure 4 The adjusted RSP pathway for large teat after reclassifying "Trace" to "Uncertain".

Notes: The category (blue) is based on milk temperature in ${ }^{\circ} \mathrm{C}$ (yellow) for large bottle teat. The end results are given in green. "Trace" outcome category reclassification to "Uncertain" in the case of $<4 \mathrm{~L}$ milk consumption.

Abbreviation: RSP, response surface pathway.

unexpected or new findings during clinical trials. In many clinical trials, for instance when clear cut interpretation of clinical outcomes is complex, use of an odd outcome category "Uncertain" between "Yes" and "No" could improve the study design flexibility. In the present study, we used this developed RSP methodology to handle odd outcomes coupled with an odd number of patient-increase procedure. The obtained results with no X-ray findings in any of the 30 feedings of the 15 calves indicate a large statistical outcome accuracy.

Regarding the recorded odd outcomes, the results show interesting opportunities for how the RSP method can be further developed to handle study outcome uncertainty. Lack of X-ray findings in any of the feedings without considering the uncertainty arising from the $4 \mathrm{~L}$ milk consumption deviations introduces new questions around interpretation of the present study outcomes. In six of the 30 feedings, the calves did not manage to drink all the prescribed milk. Regardless of the many potential physiological reasons for and relevance of this protocol deviation, it exposes an observed clinical finding that needs to be addressed. Reclassification of the category "Trace" to "Uncertain" includes the uncertainty around the observed $<4 \mathrm{~L}$ milk consumption. This unlocks an alternative RSP pathway and is one way of addressing uncertainty of achieved outcomes in hindsight. Analyzing how an outcome 
category reclassification alters the study results can be done by walking adjusted outcomes down the RSP map. In the present study, the estimated MMT after category reclassification to include $<4 \mathrm{~L}$ milk consumption kept the study result to $8^{\circ} \mathrm{C}$ for small teat, but altered it for large teat to $14.3^{\circ} \mathrm{C}$. A second look at study results after reclassification of clinical results might be valuable and introduce flexibility in many clinical trials.

Maneuvering one continuous outcome variable like milk temperature or drug dose window in RSP together with odd number of ordinal outcomes like yes/trace/no or yes/uncertain/no requires a randomization procedure between design levels. For RSP, the three temperature outcomes $\mathrm{a}_{1-3}$ in design level 1 indicates at the next dose k-factor-adjusted temperature $\mathrm{A}_{1-3}$ to undergo randomization and they are selected to pinpoint the respective five temperatures clinically used in design level 2. Similarly, the three temperature-adjusted $A_{1-3}$ for small and large teats, respectively, give rise to the five next temperatures which in turn in design level 2 produce the clinical outcomes $b_{1-5}$. Furthermore, the five temperature outcomes in design level 2 will undergo randomization and in turn point out the selected pathway for the seven next temperatures to be used in design level 3. This randomization procedure was found appropriate in the present material.

The additional RSP methodology procedures introduced in this trial also include combination with LSQ study design to compare the influence of small and large teats for milk feeding. Despite an unbalanced $2 \times 2$ LSQ in the present study, the use of RSP in each LSQ teat sequence can give increased flexibility, reduced sample size, and new opportunities in RCT compared to alternative study design approaches. An improvement of this combined RSP-LSQ study design could be to use an even number of included patients. By changing the number of patients at the three design levels from $3,5,7$ to $2,4,8$, the present unbalanced $2 \times 2$ LSQ could be performed balanced and hence increase the power of the study. In the present study, the skewness due to the odd number of included subjects may only give a small and limited influence on the results, but including an even number of subjects would improve the RSP-LSQ design.

The combination of the randomization performed separately for small and large bottle teats may have been developed differently. Due to the use of LSQ in the present study, randomization between small and large teats to give rise to three different temperature-teat combinations. These combinations were used as basis for allocation of the five calves in the second design level. By the same strategy, the five calves in design level 2 give rise to five combinations for allocation in design level 3. In this way, both milk temperature and teat size are randomized between each study design level, each individual calf serving as its own control in the LSQ teat sequence, creating a combined randomized RSP in a LSQ designed trial.

Reduction in sample size while increasing statistical accuracy is an important goal for developing and improving clinical trial methods. ${ }^{5,6}$ Stipulating outcome accuracy and calculating sample size in alternative study designs with the same study aims will of course be influenced by the chosen model. From both an ethical and statistical point of view, the sample size must be calculated in the planning stage of the study. In the present $2 \times 2$ LSQ design, the number of included subjects for each outcome category would have to be 12 or 18 to obtain clinically relevant differences within a $95 \%$ or $99 \%$ power. ${ }^{18}$ Dividing the temperature window into conservative $5^{\circ} \mathrm{C}$ incremental temperature intervals would give six outcome categories. To cover all these categories in a classical $2 \times 2$ LSQ design with a significance level of $5 \%$ and a power of $95 \%$ would require inclusion of $12 \times 6=72$ subjects. Furthermore, a significance level of $1 \%$ and a power of $99 \%$ would require inclusion of 108 subjects in a traditional $2 \times 2$ LSQ design. Even though it is difficult to quantify the outcome accuracy differences between study designs without simulations, this comparison indicates statistical and clinical superiority of the RSP-LSQ combination over a traditional LSQ strategy.

Combination of RSP in an LSQ designed study for maneuvering one continuous and one discrete ordinal outcome variable demonstrates how RSP can be developed to an RCT. Inclusion of two or more interventional variables in higher dimensional RSP designs, maneuvering continuous outcome variables for instance in dose finding studies, could further improve the methodology.

\section{Conclusion}

The randomized between-patient RSP in an LSQ-designed RCT detected its power and predicted the outcome variable with high accuracy. The study demonstrates advantages of RSP methodology with increasing patient number along with increasing design levels, use of odd number of outcomes to handle uncertainty, randomization procedure between design levels, and use of RSP in an LSQ designed study.

\section{Acknowledgments}

The authors would like to thank clinicians and staff members at the Norwegian University of Life Science, Faculty of Veterinary Science, Department of Production Animal Clinical 
Sciences and the Norwegian Veterinary Institute for their participation in the study. The data sets used and analyzed during the current study are available from the corresponding author on reasonable request. The study was funded by the Norwegian University of Life Science, Faculty of Veterinary Science, Department of Production Animal Clinical Sciences, Centre for Epidemiology and Biostatistics and the Norwegian Veterinary Institute, Oslo, Norway.

\section{Author contributions}

KE was responsible for study protocol, clinical trial conduct, $\mathrm{X}$-ray interpretations, and data gathering. SD performed the methodological response surface pathway (RSP) review interpretation and gave suggestions for improvements in the dose adjustment procedures. TH performed the overall manuscript write up, RSP methodological development and descriptions. SL developed, analyzed, and interpreted the statistical procedures for the RSP methodology. All authors contributed toward data analysis, drafting and critically revising the paper and agree to be accountable for all aspects of the work.

\section{Disclosure}

The authors report no conflicts of interest in this work.

\section{References}

1. Sjaastad OV, Sand O, Hove K. Physiology of Domestic Animals. 2nd ed. Oslo: Scandinavian Veterinary Press; 2010:804. ISBN: 9788-2917-4397-3.

2. Lateur-Rowet HJ, Breukink HJ. The failure of the esophageal groove reflex, when fluids are given with an esophageal feeder to newborn and young calves. Vet $Q .1983 ; 5: 68-74$.
3. Swanson EW, Harris JD. Development of rumination in the young calf. J Dairy Sci. 1958;41:1768-1776.

4. Ellingsen K, Mejdell C, Ottesen N, Larsen S, Grøndahl AM. The effect of large milk meals on digestive physiology and behavior in dairy calves. Physiol Behav. 2016;154:169-174.

5. Hulley SB, Cummings SR, Browner SW, Grady DG, Newman TB. Designing Clinical Research. Philadelphia, PA: Lippincott Williams \& Wilkins; 2013. ISBN: 9781608318049.

6. Saccà L. The uncontrolled clinical trial: scientific, ethical, and practical reasons for being. Intern Emerg Med. 2010;5(3):201-204.

7. Stylianou M, Flournoy N. Dose finding using the biased coin upand-down design and isotonic regression. Biometrics. 2002;58(1): 171-177.

8. Aune T, Sorby R, Yasumoto T, Ramstad H, Landsverk T. Comparison of oral and intraperitoneal toxicity of yessotoxin towards mice. Toxicon. 2002;40(1):77-82.

9. Ryeng KA, Arnemo JM, Larsen S. Determination of optimal immobilizing doses of medetomidine-ketamine in captive reindeer (Rangifer tarandus tarandus). An iteration-designed controlled clinical study in wildlife medicine. Am J Vet Res. 2001;62:119-126.

10. Dewi S, Kristiansen VM, Lindkær-Jensen S, Larsen S. Between- and within-patient, $n$-level response surface pathway design in dose-finding studies. Open Access J Clin Trials. 2014;6:63-74.

11. Dewi S, Aune T, Buanæs JAA, Smith AJ, Larsen S. The development of response surface pathway design to reduce animal numbers in toxicity studies. BMC Pharmacol Toxicol. 2014;15:18.

12. Altman DG. Randomisation. BMJ. 1991;302:1481-1482.

13. Kendall JM. Designing a research project: randomised controlled trials and their principles. Emerg Med J. 2003;20:164-168.

14. Dewi S, Larsen S, Srimuninnimit V, LuYS, Manuaba T, Lindkær-Jensen S. Benzene-poly-carboxylic acids complex with cis-diammineplatinum (II) dichloride in the treatment of stage IV breast cancer patients. Open Breast Cancer J. 2013;5:7-15.

15. Fisher G. Series and Sequences. Putney: Phoenix Education; 1996.

16. Paul RK, Rosenberger WF, Flournoy N. Quantile estimation following non-parametric phase I clinical trials with ordinal response. Stat Med. 2004;23(16):2483-2495.

17. Altman DG. Practical Statistic for Medical Research. London: Chapman \& Hall; 1991. ISBN-13: 978-0412276309.

18. Larsen S, Osnes M, Eidsaunet W, Sandvik L. Factors influencing the sample size, exemplified by studies on gastroduodenal tolerability of drugs. Scand J Gastroenterol. 1985;20(4):395-400.
Open Access Journal of Clinical Trials

\section{Publish your work in this journal}

The Open Access Journal of Clinical Trials is an international, peerreviewed, open access journal publishing original research, reports, editorials, reviews and commentaries on all aspects of clinical trial design, management, legal, ethical and regulatory issues, case record form design, data collection, quality assurance and data auditing

\section{Dovepress}

methodologies. The manuscript management system is completely online and includes a very quick and fair peer-review system, which is all easy to use. Visit http://www.dovepress.com/testimonials.php to read real quotes from published authors. 